\title{
Concordant peripheral lipidome signatures in two large clinical studies of Alzheimer's disease
}

\author{
Kevin Huynh (1) 1,2,28, Wei Ling Florence Lim (10 3,4,28, Corey Giles ${ }^{1}$, Kaushala S. Jayawardana ${ }^{1}$, Agus Salim 1,5,6,7, \\ Natalie A. Mellett ${ }^{1}$, Adam Alexander T. Smith1, Gavriel Olshansky1, Brian G. Drew ${ }^{1,2}$, \\ Pratishtha Chatterjee (iD ${ }^{3,8,9}$, lan Martins (iD) ${ }^{3,4}$, Simon M. Laws (D) 3,10,11, Ashley I. Bush (D) ${ }^{12}$, \\ Christopher C. Rowe ${ }^{12,13}$, Victor L. Villemagne ${ }^{13,14}$, David Ames ${ }^{15}$, Colin L. Masters ${ }^{12}$, Matthias Arnold (1) ${ }^{16,17}$, \\ Kwangsik Nho ${ }^{18,19,20}$, Andrew J. Saykin (10) 18,20,21, Rebecca Baillie (1) 22, Xianlin Han (D) ${ }^{23}$, \\ Rima Kaddurah-Daouk (16,24,25,29凶, Ralph N. Martins ${ }^{3,4,8,9,26,27,29 凶}$ \& Peter J. Meikle (1) 1,2,29凶
}

Changes to lipid metabolism are tightly associated with the onset and pathology of Alzheimer's disease (AD). Lipids are complex molecules comprising many isomeric and isobaric species, necessitating detailed analysis to enable interpretation of biological significance. Our expanded targeted lipidomics platform ( 569 species across 32 classes) allows for detailed lipid separation and characterisation. In this study we examined peripheral samples of two cohorts (AIBL, $n=$ 1112 and $A D N I, n=800$ ). We are able to identify concordant peripheral signatures associated with prevalent $A D$ arising from lipid pathways including; ether lipids, sphingolipids (notably $G_{3}$ gangliosides) and lipid classes previously associated with cardiometabolic disease (phosphatidylethanolamine and triglycerides). We subsequently identified similar lipid signatures in both cohorts with future disease. Lastly, we developed multivariate lipid models that improved classification and prediction. Our results provide a holistic view between the lipidome and $A D$ using a comprehensive approach, providing targets for further mechanistic investigation.

\footnotetext{
${ }^{1}$ Baker Heart and Diabetes Institute, Melbourne, VIC, Australia. ${ }^{2}$ Monash University, Melbourne, VIC 3800, Australia. ${ }^{3}$ School of Medical and Health Sciences, Edith Cowan University, Perth, WA, Australia. ${ }^{4}$ Cooperative research Centre (CRC) for Mental Health, Sydney, NSW, Australia. ${ }^{5}$ Department of Mathematics and Statistics, La Trobe University, Melbourne, VIC, Australia. ${ }^{6}$ Melbourne School of Global and Population Health, The University of Melbourne, Melbourne, VIC 3010, Australia. ${ }^{7}$ School of Mathematics and Statistics, The University of Melbourne, Melbourne, VIC 3010, Australia. ${ }^{8}$ Department of Biomedical Sciences, Macquarie University, Sydney, NSW, Australia. ${ }^{9}$ KaRa Institute of Neurological Disease, Sydney, NSW, Australia.

${ }^{10}$ Collaborative Genomics Group, School of Medical and Health Sciences, Edith Cowan University, Perth, WA, Australia. ${ }^{11}$ Curtin Health Innovation Research Institute, Curtin University, Perth, WA, Australia. ${ }^{12}$ The Florey Department of Neuroscience and Mental Health, The University of Melbourne, Melbourne, VIC, Australia. ${ }^{13}$ Department of Nuclear Medicine and Centre for PET, Austin Health, Heidelberg, VIC, Australia. ${ }^{14}$ Department of Medicine, Austin Health, The University of Melbourne, Heidelberg, VIC, Australia. ${ }^{15}$ National Ageing Research Institute, Parkville, VIC 3050 , Australia. ${ }^{16}$ Department of Psychiatry and Behavioral Sciences, Duke University, Durham, NC, USA. ${ }^{17}$ Institute of Computational Biology, Helmholtz Zentrum München, German Research Center for Environmental Health, Neuherberg, Germany. ${ }^{18}$ Department of Radiology and Imaging Sciences, Indiana University School of Medicine, Indianapolis, IN, USA. ${ }^{19}$ Center for Computational Biology and Bioinformatics, Indiana University School of Medicine, Indianapolis, IN, USA. ${ }^{20}$ Indiana Alzheimer Disease Center, Indiana University School of Medicine, Indianapolis, IN, USA. ${ }^{21}$ Department of Medical and Molecular Genetics, Indiana University School of Medicine, Indianapolis, IN, USA. ${ }^{22}$ Rosa \& Co LLC, San Carlos, CA, USA. ${ }^{23}$ Barshop Institute for Longevity and Aging Studies, University of Texas Health Science Center at San Antonio, San Antonio, TX, USA. ${ }^{24}$ Duke Institute of Brain Sciences, Duke University, Durham, NC, USA. ${ }^{25}$ Department of Medicine, Duke University, Durham, NC, USA. ${ }^{26}$ School of Psychiatry and Clinical Neurosciences, The University of Western Australia, Perth, WA, Australia. 27 Australian Alzheimer's Research Foundation, Nedlands, WA, Australia. ${ }^{28}$ These authors contributed equally: Kevin Huynh, Wei Ling Florence Lim. ${ }^{29}$ These authors jointly supervised this work: Rima Kaddurah-Daouk, Ralph N. Martins, Peter J. Meikle. ${ }^{凶}$ email: rima.kaddurahdaouk@duke.edu; r.martins@ecu.edu.au; peter.meikle@baker.edu.au
} 
lzheimer's disease $(\mathrm{AD})$ is a neurodegenerative disease characterised by progressive decline in cognitive function, usually presenting with memory loss. In the sporadic form of $\mathrm{AD}$, symptoms usually begin to manifest after the age of 65 , and with the ageing global population, the number of people with $\mathrm{AD}$ has been estimated to reach 81 million worldwide by 2040 (ref. ${ }^{1}$ ). The failure of many AD clinical trials over recent years has led to the call for a paradigm shift in $\mathrm{AD}$ research. It is now recognised that additional underlying mechanisms are involved in the pathogenesis of $\mathrm{AD}$. We seek to provide a deeper molecular understanding of metabolic pathways implicated in $\mathrm{AD}$ to identify key enzymes, transporters and signalling molecules that are most amenable for therapeutic targeting. As there are no appropriate sporadic mouse models of $\mathrm{AD}$, human studies are essential for better understanding pathogenesis of $\mathrm{AD}$. In particular, statistically powered studies are needed to detect the associations beneath the natural human biological variation.

Lipids are fundamental to every living system. These diverse and biologically important molecules comprise thousands of individual species, spanning multiple classes and subclasses. In plasma, the majority of lipids are small amphiphilic molecules (including cholesterol) that make up the circulating lipoprotein particles such as high- and low-density lipoprotein (HDL and LDL). With recent advances to mass spectrometry and highperformance liquid chromatography, it is now feasible to examine in detail the comprehensive plasma lipidome in a human population or clinical study ${ }^{2}$. Quantification and characterisation of these diverse lipid molecules form the foundation of the field known as lipidomics.

Evidence that lipids are involved in AD have been demonstrated via alterations observed in phospholipid ${ }^{3-5}$, plasmalogens $^{6}$, ceramide ${ }^{7}$, ganglioside ${ }^{8}$ and sulfatide ${ }^{7,9}$ compositions in the brain. Several recent studies indicate that altered phospholipid metabolism associated with $\mathrm{AD}$ pathogenesis is also observed in the blood $5,10,11$, thus encouraging discovery studies for bloodbased lipid markers. Furthermore, a recent large-scale genomewide association meta-analysis has identified genes involved in lipid metabolism as key risk factors for $\mathrm{AD}^{12}$.

The plasma lipidome is complex and consists of many isomeric and isobaric species ${ }^{13}$, these are species that share similar or identical elemental composition but might be structurally different and display specific associations with biological outcomes. Existing lipidomic studies often employ techniques that either have limited coverage of the lipidome (e.g., focusing on one or two lipid classes) and/or provide poor resolution of lipid species $^{5,14-17}$. Limited specificity of lipidomic platforms can result in the aggregation of multiple species in one signal, limiting interpretation and reproducibility. Comprehensive approaches using untargeted lipidomics can provide greater coverage of the lipidome ${ }^{18,19}$, but can sometimes result in ambiguous identifications. More recent studies into lipidomics and dementia with updated methodology have shown that more structural granularity can lead to improved interpretation of results ${ }^{20}$. We have recently expanded our lipidomic platform to better characterise isomeric lipid species, now measuring 569 lipids from 32 classes and subclasses. Our methodology focuses on lipid and lipid-like compounds utilising chromatographic separation. We have applied this methodology to two large independent studies: The Australian Imaging, Biomarkers and Lifestyle (AIBL) flagship study of ageing ${ }^{21}$ and the Alzheimer's Disease Neuroimaging Initiative baseline (ADNI) cohort. Here we show the importance of capturing the comprehensive lipidome and highlight the necessity of obtaining molecular structural detail to identify key lipid pathways to link the plasma lipidome with $\mathrm{AD}$ and the future onset of $\mathrm{AD}$.

\section{Results}

Lipidomic analysis of the AIBL and ADNI cohort. Between the two cohorts, a total of 5733 samples (including quality controls and blanks) on 1912 unique individuals were analysed. The characteristics of individuals in the cross-sectional and longitudinal analysis are shown in Table 1 and further breakdowns are provided in Supplementary Table 1.

We developed our platform to better characterise the lipids in human plasma ${ }^{22}$. In total, we are able to examine 32 lipid classes and subclasses (conditions detailed in Supplementary Table 2) for both cohorts. In general, a similar correlation structure was observed within the AIBL and ADNI cohorts between lipid classes and many clinical measures (Fig. 1). Many commonly reported lipid species associated with $\mathrm{AD}$, such as sphingolipids and ether lipids, are often reported with ambiguous annotations, where the reported species are the sum of several isomers. Here we report the detailed characterisation of these species.

Sphingolipids are structurally resolved through collisioninduced dissociation (CID), where fragments correspond to the sphingoid base, with the exception of sphingomyelins. Dissociation of sphingomyelin species under normal conditions results in a product ions that yields only sum composition data, i.e. the sphingomyelin species, $\operatorname{SM}(42: 2)$. To determine sphingomyelin structural composition, we repeated the mass spectrometry analysis on pooled plasma samples in the presence of lithium acetate as described previously ${ }^{22}$. The lithiated adduct of sphingomyelins produces product ions corresponding to the sphingoid base and $n$-acyl chain (Fig. 1a) allowing for structural identification. Alignment through chromatography highlights that our measurement of $\operatorname{SM}(42: 2)$, for example, is chromatographically separated into $\mathrm{SM}(\mathrm{d} 18: 1 / 24: 1)$ and $\mathrm{SM}(\mathrm{d} 18: 2 / 24: 0)$ (Fig. 1a).

Similarly, this approach was repeated with the different glycerophospholipid classes to capture isomeric and isobaric structural details where they were chromatographically resolved. Examination of the transition $\mathrm{m} / z$ 770.6/184.1 corresponding to the phosphatidylcholine/alkylphosphatidylcholine/alkenylphosphatidylcholine species $\mathrm{PC}(35: 3) / \mathrm{PC}(\mathrm{O}-36: 3) / \mathrm{PC}(\mathrm{P}-36: 2)$ results in nine distinct peaks (Fig. 1b). Here we report the complete separation of diacyl odd-numbered phosphatidylcholine species, the non-plasmalogen ether lipids $\mathrm{PC}(\mathrm{O})$ and the plasmalogen ether lipids, $\mathrm{PC}(\mathrm{P})$. Results were confirmed by exploiting the susceptibility of plasmalogens to acid hydrolysis (Fig. 1b). The correlation structure of all 32 classes and subclasses along with clinical variables is depicted in Fig. 1c.

Concordance of associations between two studies with AD. After adjustment for covariates (including age, sex, body mass index (BMI), total cholesterol, HDL-C, triglycerides, site of sample collection, APOE $\varepsilon 4$ alleles, omega- 3 supplementation and statin use). There were 12 and 3 classes significantly associated with $A D$ in the AIBL and ADNI1 cohorts, respectively, after false discovery rate (FDR) correction (Fig. 2), corresponding to 147 and 87 lipids, respectively (219 and 157 uncorrected, Fig. 3). Meta-analysis using a fixed-effects model identified 197 lipids and 11 classes associated with $\mathrm{AD}$ between both cohorts (Figs. 2 and 3). The lipid classes associated were predominately from the sphingolipid classes: dihydroceramides (dhCer), trihexosylceramides (Hex3Cer), $\mathrm{GM}_{3}$ gangliosides $\left(\mathrm{GM}_{3}\right), \mathrm{GM}_{1}$ gangliosides $\left(\mathrm{GM}_{1}\right)$ and ether lipids classes: alkylphosphatidylcholine $[\mathrm{PC}(\mathrm{O})]$, alkenylphosphatidylcholine $[\mathrm{PC}(\mathrm{P})]$, alkylphosphatidylethanolamine $[\mathrm{PE}(\mathrm{O})]$, alkenylphosphatidylethanolamine $[\mathrm{PE}(\mathrm{P})]$, alkyldiacylglycerol [TG(O)].

While all plasmalogens (alkenyl classes) are ether lipids, not all ether lipids are plasmalogens. This distinction is important when factoring in their biological significance. Independent of multiple 
Table 1 Study characteristics.

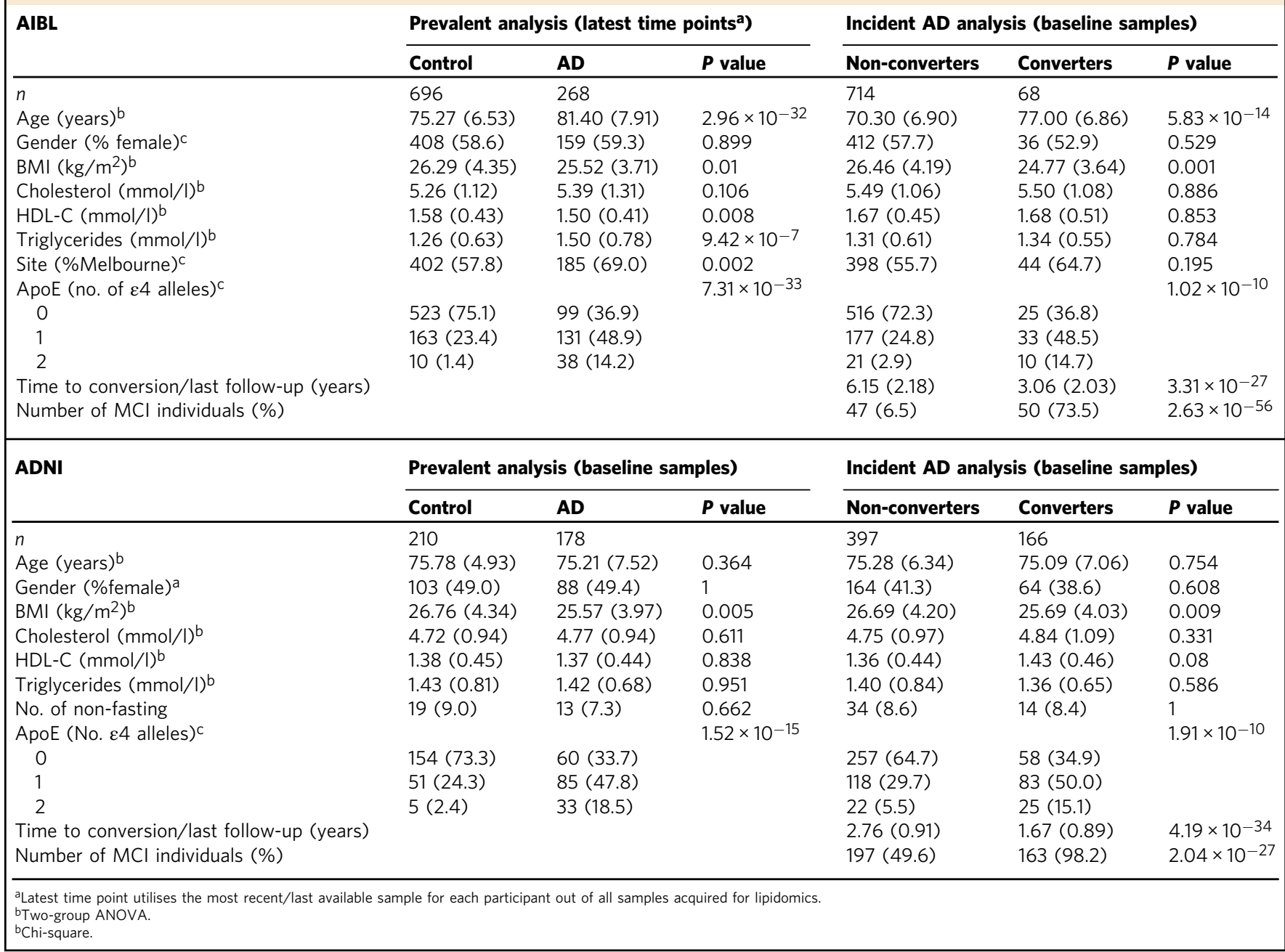

covariates, the majority of the ether lipid classes were negatively associated with AD (Figs. 2 and 3a). This effect is compounded when the ether lipids are esterified with omega-3 fatty acids, such as the 22:6 acyl chain (docosahexaenoic acid, DHA). However, it should be noted that ether lipids with polyunsaturated fatty acids other than omega-3 were still negatively associated with AD (Fig. 3b and Supplementary Table 3), highlighting that the effect is not necessarily driven by omega-3 fatty acids. A nonglycerophospholipid subclass of ether lipids, alkyldiacylglycerols, $\mathrm{TG}(\mathrm{O})$, were negatively associated with $\mathrm{AD}$, despite the species measured predominately containing saturated or monounsaturated species (Figs. 2 and 3 ).

Ceramide and sphingomyelin species presented with both positive and negative associations with $\mathrm{AD}$ resulting in no significant associations at the class level. In both cohorts, dhCer was notability negatively associated with $\mathrm{AD}$. Negative associations were primarily driven by 22:0 and 24:0 saturated species in the sphingolipid $n$-acyl moiety, while positive associations were predominately the shorter 18:0, 20:0 and monounsaturated 24:1 species. The sphingoid base had no apparent influence on the association in AD. This sphingolipid pattern in AD is much weaker in the ADNI1 cohort (Fig. 3 and Supplementary Table 3).

This effect was also seen for other complex sphingolipids, where a positive association or trend was observed at the class level (monohexosylceramide, HexCer, dihexosyolceramide, Hex2Cer, Hex3Cer, $\mathrm{GM}_{3}$ and $\mathrm{G}_{\mathrm{M} 1}$ gangliosides) in both $\mathrm{AIBL}$ and
ADNI cohorts (Fig. 2), but individual species within these classes present with the same opposing relationship (negative association with the $n$-acyl chains 22:0 and 24:0, and positive association with the 18:0, 20:0 and 24:1 species). This combined trend resulted in no association with 22:0 and 24:0 $n$-acyl sphingolipids but an increased association in sphingolipids with $n$-acyl chains 18:0, 20:0 and 24:1, such as $\mathrm{GM}_{3}(\mathrm{~d} 18: 1 / 24: 1)$ which has the strongest positive association with AD (Fig. 3 and Supplementary Table 3).

A notable pattern observed in both AIBL and ADNI cohorts, even after adjustment for clinical measures of cholesterol and triglycerides (TGs), was the positive associations of the lipid classes phosphatidylethanolamine (PE) and TG (Fig. 3). This effect has been noted in diseases where dyslipidemia is prevalent such as type 2 diabetes $^{23}$.

Similar lipids are associated with cross sectional and longitudinal analysis of $\mathrm{AD}$ in both cohorts. Using Cox regression models, we explored lipids associated with the risk of developing $\mathrm{AD}$ in the future. Baseline characteristics of this analysis are presented in Supplementary Table 1. We noted that in both cohorts, there was also a higher proportion of individuals with mild cognitive impairment (MCI) in the conversion group. After adjustment for covariates (age as time scale, sex, BMI, total cholesterol, HDL-C, TGs, site of sample collection, APOE $\varepsilon 4$ alleles, omega-3 supplementation and statin use), there were 
a

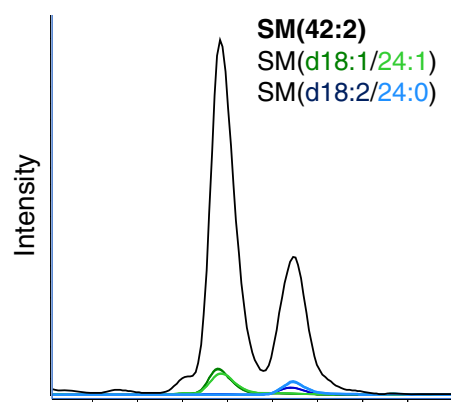

Retention time

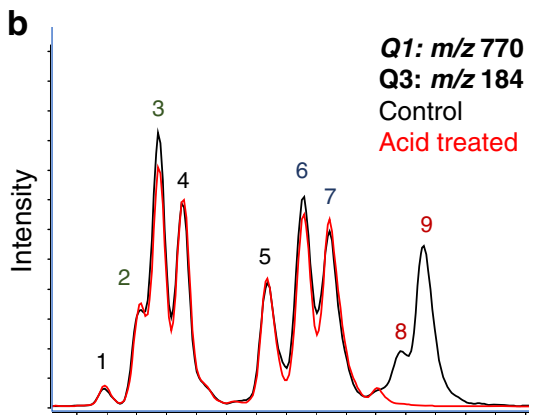

Retention time
1) +2 isotope of $\mathrm{PC}(35: 4)$

2) $P C(35: 3)(a)$

3) $\operatorname{PC}(35: 3)(b)$

4) +1 isotope of $\mathrm{SM}(39: 3)$

5) +2 isotope of $\mathrm{PC}(\mathrm{O}-36: 4)$

6) $\mathrm{PC}(\mathrm{O}-36: 3)(\mathrm{a})$

7) $\mathrm{PC}(\mathrm{O}-36: 3)$ (b)

8) $\mathrm{PC}(\mathrm{P}-36: 2)(\mathrm{a})$

9) $\mathrm{PC}(\mathrm{P}-36: 2)(\mathrm{b})$

C

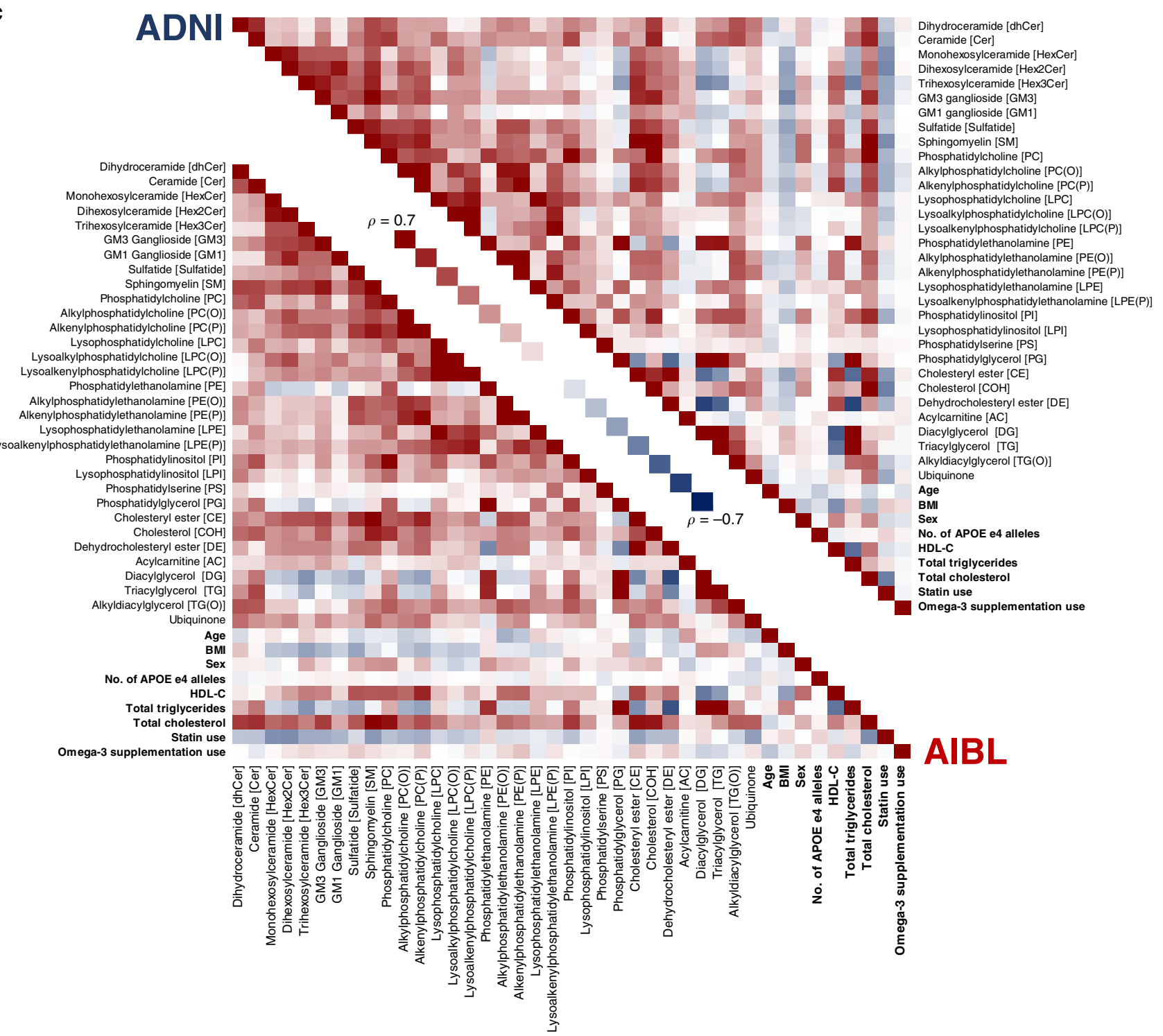

Fig. 1 Characterisation of lipid isomeric species and the relationship of lipid classes and subclasses within the AIBL and ADNI cohorts.

a Characterisation of sphingomyelin isomers. Black trace corresponds to the chromatogram seen under normal conditions. Additional experimental results in the green and blue traces used for identification, corresponding to SM(d18:1/24:1) and SM(d18:2/24:0) respectively. b Characterisation of glycerophospholipid isomers. Black trace corresponds to the chromatogram seen under normal conditions. Red trace is the same scan after sample acid hydrolysis. c Spearman correlation of total lipid classes, subclasses and commonly reported clinical measures (bolded) for the AIBL baseline and ADNI studies. 
a

Dihydroceramide [dhCer]

Ceramide [Cer]

Monohexosylceramide [HexCer]

Dihexosylceramide [Hex2Cer]

Trihexosylceramide [Hex3Cer]

GM3 ganglioside [GM3]

GM1 ganglioside [GM1]

Sulfatide [Sulfatide]

Sphingomyelin [SM]

Phosphatidylcholine $[\mathrm{PC}]$

Alkylphosphatidylcholine [PC(O)

Alkenylphosphatidylcholine $[\mathrm{PC}(\mathrm{P})]$

Lysophosphatidylcholine [LPC]

Lysoalkylphosphatidylcholine [LPC(O)]

Lysoalkenylphosphatidylcholine [LPC(P)]

Phosphatidylethanolamine [PE]

Alkylphosphatidylethanolamine [PE(O)]

Alkenylphosphatidylethanolamine $[\mathrm{PE}(\mathrm{P})]$

Lysophosphatidylethanolamine [LPE]

Lysoalkenylphosphatidylethanolamine $[\mathrm{LPE}(\mathrm{P})]$

Phosphatidylinositol [PI]

Lysophosphatidylinositol [LPI]

Phosphatidylserine [PS]

Phosphatidylglycerol [PG]

Cholesteryl ester [CE]

Cholesterol [COH]

Dehydrocholesteryl ester [DE]

Acylcarnitine $[\mathrm{AC}]$

Diacylglycerol [DG]

Triacylglycerol [TG]

Alkyldiacylglycerol [TG(O)]

Ubiquinone

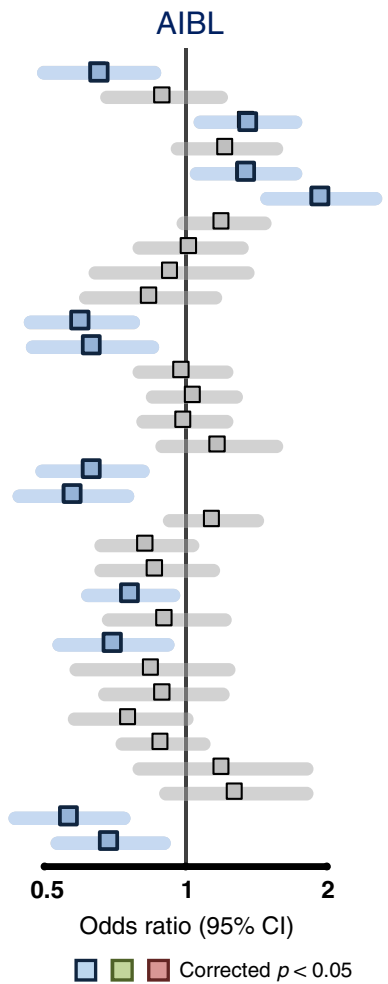

b

Dihydroceramide [dhCer]

Ceramide [Cer]

Monohexosylceramide [HexCer]

Dihexosylceramide [Hex2Cer]

Trihexosylceramide [Hex3Cer]

GM3 ganglioside [GM3]

GM1 ganglioside [GM1]

Sulfatide [Sulfatide]

Sphingomyelin [SM]

Phosphatidylcholine [PC]

Alkylphosphatidylcholine [PC(O)]

Alkenylphosphatidylcholine $[\mathrm{PC}(\mathrm{P})]$

Lysophosphatidylcholine [LPC]

Lysoalkylphosphatidylcholine [LPC(O)]

Lysoalkenylphosphatidylcholine [LPC(P)]

Phosphatidylethanolamine $[\mathrm{PE}]$

Alkylphosphatidylethanolamine $[\mathrm{PE}(\mathrm{O})]$

Alkenylphosphatidylethanolamine $[\mathrm{PE}(\mathrm{P})]$

Lysophosphatidylethanolamine [LPE]

Lysoalkenylphosphatidylethanolamine [LPE(P)]

Phosphatidylinositol [PI]

Lysophosphatidylinositol [LPI]

Phosphatidylserine [PS]

Phosphatidylglycerol [PG]

Cholesteryl ester [CE]

Cholesterol [COH]

Dehydrocholesteryl ester [DE]

Acylcarnitine $[\mathrm{AC}]$

Diacylglycerol [DG]

Triacylglycerol [TG]

Alkyldiacylglycerol [TG(O)

Ubiquinone

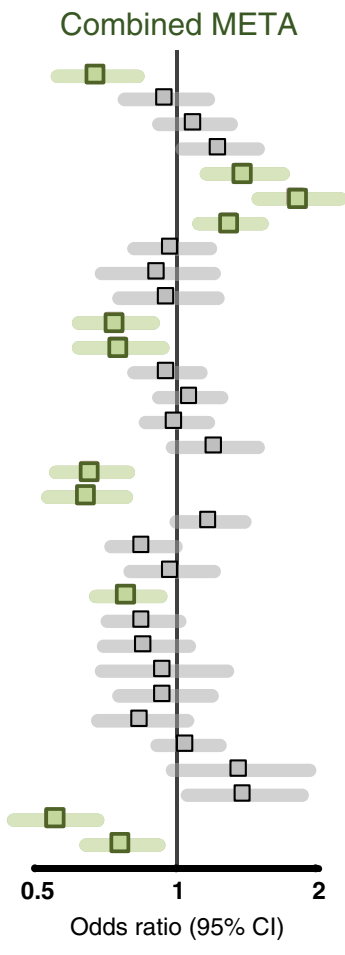

$\square$ Uncorrected $p<0.01$

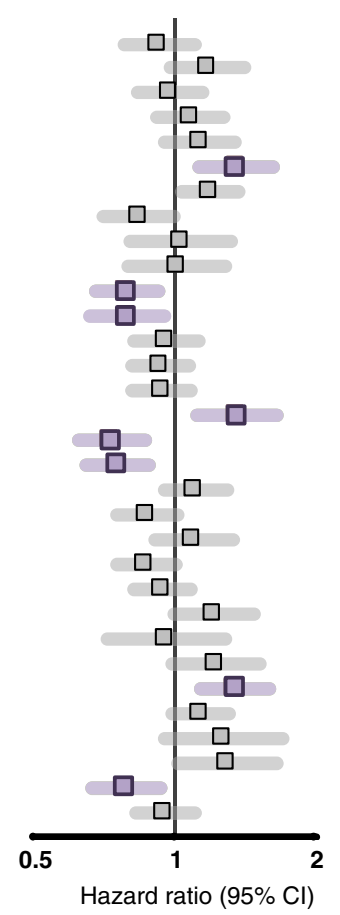

Hazard ratio $(95 \% \mathrm{Cl})$

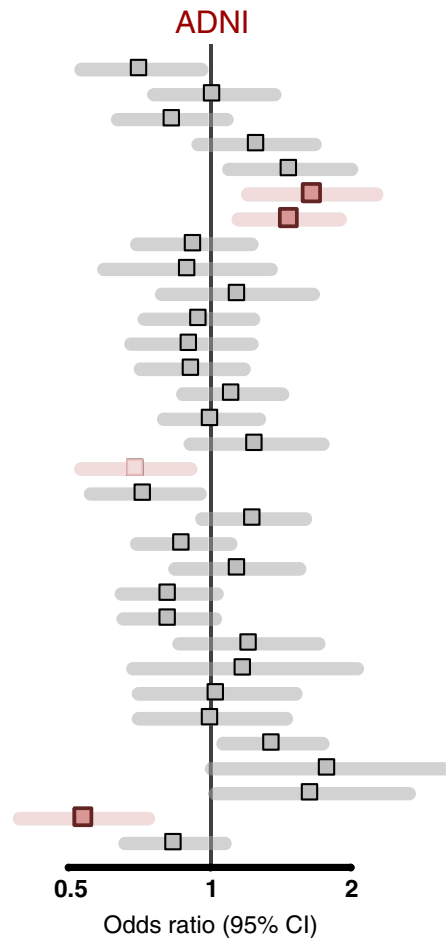

$\square$ Uncorrected $p>0.01$

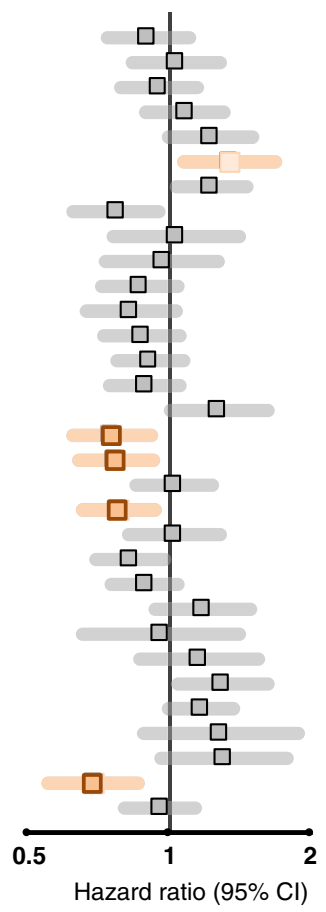

$\square$ Uncorrected $p>0.01$

Fig. 2 Associations of lipid class totals with prevalent and incident Alzheimer's disease. Forest plots of lipid class associations for a prevalent Alzheimer's disease (logistic regression, AIBL $=268$ cases, 696 control, ADNI = 178 cases, 210 controls) and $\mathbf{b}$ incident Alzheimer's disease (Cox regression, $\mathrm{AIBL}=68$ cases, 714 controls, $\mathrm{ADNI}=166$ cases, 397 controls). Lipid classes are generated by the sum of each individual species measured in each class. Regressions are adjusted for age, sex, BMI, total cholesterol, HDL-C, triglycerides, number of APOE4 alleles, statin use and omega-3 supplementation. AIBL was further adjusted for time points (only in logistic regression analysis) and site of blood collection. ADNI was further adjusted for fasting status. 


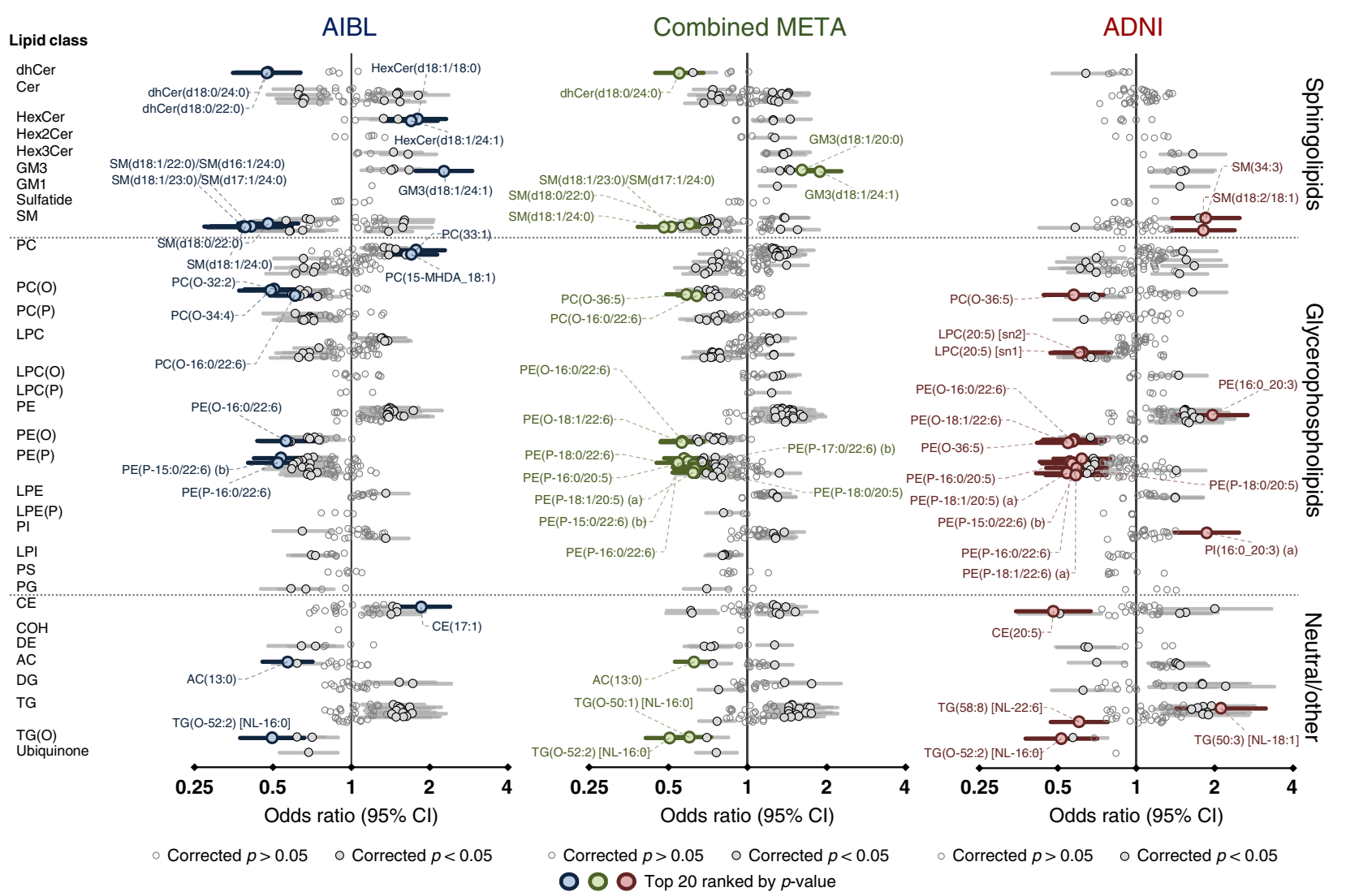

Fig. 3 Associations of individual lipid species with prevalent Alzheimer's disease. Forest plot outlining the logistic regression results of individual species, between controls and prevalent AD in both the AIBL (blue $n=268$ cases, 696 controls) and ADNI (red, $n=178$ cases, 210 controls) cohorts with the combined meta-analysis in the middle (green). P value was corrected for multiple comparison using approach by Benjamini and Hochberg. Covariates include age, sex, BMI, total cholesterol, HDL-C, triglycerides, number of APOE4 alleles, statin use and omega-3 supplementation. Additional covariates for AIBL include site of blood collection and time point while ADNI includes fasting status. Open circles, not significant; closed dark circles, significant after FDR correction; coloured circles, top 20 associations ranked by $p$ value.

71 species associated with incident $\mathrm{AD}$ in the meta-analysis (Fig. 4 and Supplementary Table 4). One hundred and sixty-one lipids had uncorrected $p$ values $<0.05$ and the majority of these show the same direction of association as observed for $\mathrm{AD}$ (Fig. 4a). These include individual species from the ether lipids, sphingolipids, PE and TG classes. If we further adjust for individuals diagnosed with $\mathrm{MCI}$, only three species remain significant after FDR correction, the sterol ester $\mathrm{DE}(18: 1)$ and two plasmalogen species.

Multivariate modelling to identify lipids important in diagnosing prevalent or predicting future onset of AD. Due to the feature selection process (selecting features based on frequency incorporating into a multivariate model), we observed that informatively prognostic lipids, but are otherwise highly correlated, would frequently be selected once in each model interchangeably, reducing their individual frequency count. We utilised the correlation data to identify lipid clusters of highly correlated species. The frequency of these clusters were then used to rank (sum of individual incorporations), with the most incorporated lipid in each cluster used as the representative for the multivariate models (Supplementary Table 5).

In the disease classification model where AIBL was the discovery set and ADNI the replication, we observed a final concordance statistic (C-statistic) of $0.752(0.747-0.757)$ through the incorporation of 10 lipid species on top of the base model of age, sex, BMI and $A P O E$ \&4 count (C-statistic of 0.731 $[0.726-0.736])$ with a Net Reclassification Index (NRI) of 0.40 when tested on the ADNI study. For incident $\mathrm{AD}$, we observed a final $C$-statistic of 0.675 (0.671-0.680), up from 0.644 (0.640-0.648) obtained from the base model alone (NRI of $0.40)$. The summary of results is presented in Table 2 .

In the parallel analysis, where the ADNI was the discovery and AIBL was the replication, the disease classification model had a final $C$-statistic of $0.869(0.866-0.871)$ through the incorporation of 10 lipid species on top of the base model of age, sex, BMI and $A P O E \varepsilon 4$ count (C-statistic of 0.820 [0.817-0.823]) with an NRI of 0.84 when tested on the AIBL study. For prediction of future disease onset, we observed a final $C$-statistic of 0.733 (0.727-0.740), up from $0.716(0.709-0.723)$ obtained from the base model alone (NRI of 0.45). The summary of results is presented in Table 2 .

\section{Discussion}

Here we present our initial results from the lipidomic analysis of the AIBL and ADNI cohorts. Our goal was to define the complex associations between lipids and AD using our updated methodology by examining each cohort independently and subsequently combining them in a single meta-analysis. The independent nature of these two studies allows for confidence in identifying lipids that are important in AD pathology.

The positive associations of $\mathrm{PE}$, diacylglycerol and triacylglycerol with $\mathrm{AD}$ is similar to the associations seen with both prediabetes and type 2 diabetes $^{23}$. These species tend to correlate closely with clinically elevated levels of TGs and reduced HDL cholesterol (dyslipidemia). Adjustment for these clinical lipid 


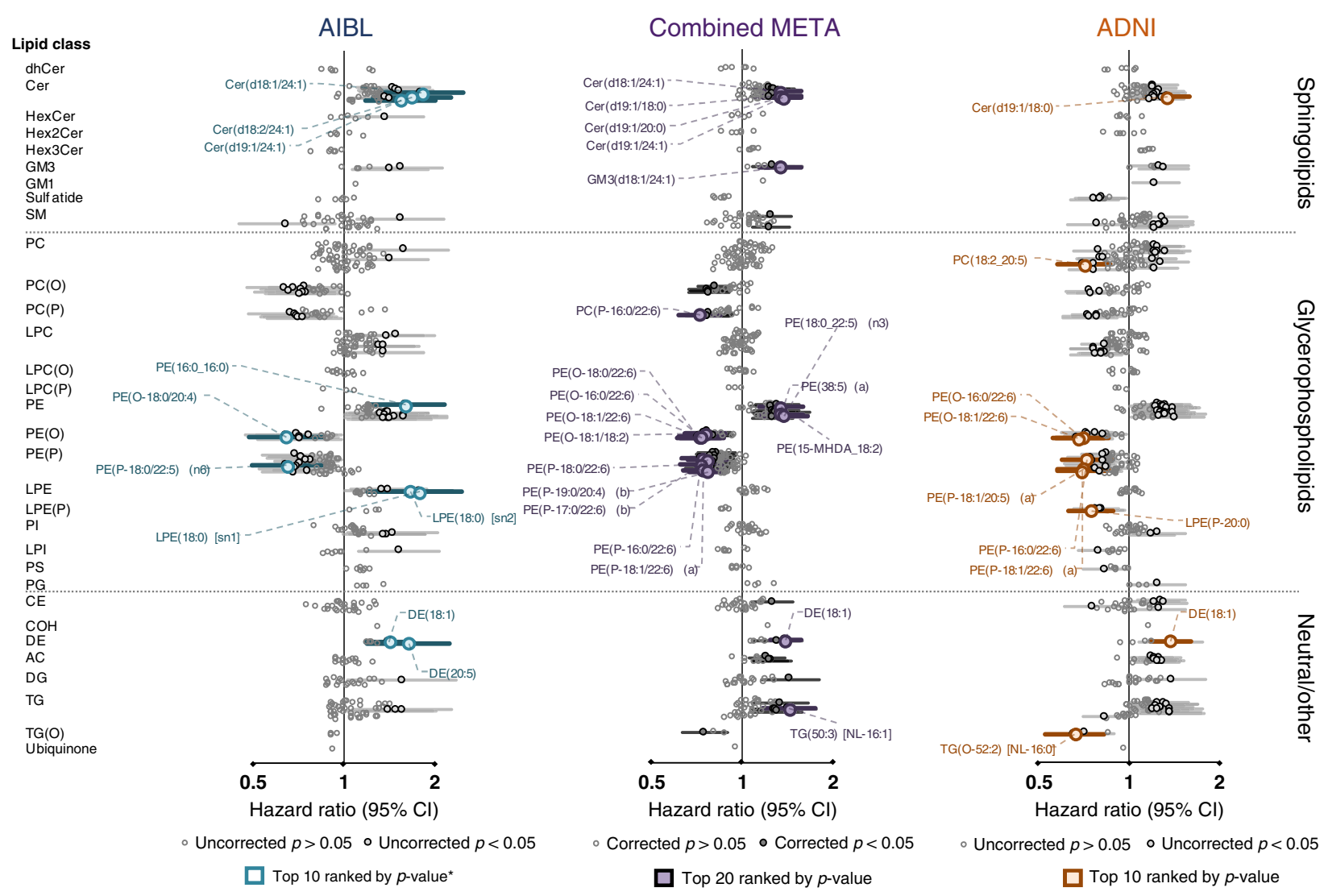

Fig. 4 Associations of individual lipid species with future onset Alzheimer's disease. Forest plot outlining the Cox regression results of individual species, between non-converters and future converters in both the AIBL (cyan $n=68$ cases, 714 controls) and ADNI (orange, $n=166$ cases, 397 controls) cohorts with the combined meta-analysis in the middle (purple). P value was corrected for multiple comparison using approach by Benjamini and Hochberg. Covariates include age (set as timescale), sex, BMI, total cholesterol, HDL-C, triglycerides, number of APOE4 alleles, statin use and omega-3 supplementation. Open circles, not significant; closed dark circles, uncorrected $p$ value; coloured squares, top 10/20 associations ranked by $p$ value.

measures resulted in a loss of significance at the class level; however, multiple species within these classes retained a significant association with AD (Fig. 3). Interestingly, we observe mostly monounsaturated acyl species driving the associations within these classes. Several studies have highlighted the association of dyslipidemia and insulin resistance with the risk of both vascular dementia and $\mathrm{AD}^{24,25}$. It may also be possible that the positive association observed with the PE class is a compensatory increase due to the decreases of $\mathrm{PE}(\mathrm{P})$ species, a phenomenon that has been reported in plasmalogen-deficient mice ${ }^{26}$.

A key difference between plasmalogens and non-plasmalogen ether lipids is the vinyl-ether bond in the $s n 1$ position, which is susceptible to oxidation ${ }^{27}$. Little of the recently reported metabolomic literature with respect to $\mathrm{AD}$ has differentiated these two classes $^{5,14-17}$. To further complicate this issue, studies which have obtained data from unit resolution mass spectrometers were unable to differentiate these species from isobaric species (odd numbered di-acyl lipids), unless extensive chromatography has also been employed. For example, $\mathrm{PC}(35: 2)$ has a mass difference of $0.036 \mathrm{Da}$ from $\mathrm{PC}(\mathrm{O}-36: 2)$ and $\mathrm{PC}(\mathrm{P}-36: 1)$, of which the latter two share identical masses (isomeric). When measured on a unitresolution instrument without chromatography ${ }^{5}$, these all contribute to the same signal. This can raise issues in instances where these species associate differently with the outcome. This is exemplified in the inverse relationship of odd-chain and ether lipids with $\mathrm{AD}$ (Supplementary Table 3), highlighting the importance of differentiating these species.

It has been proposed that plasmalogens act as endogenous antioxidants through their vinyl-ether bond and that increased oxidative stress may explain the lower levels observed in $\mathrm{AD}$ patients ${ }^{28}$. However, both plasmalogen and non-plasmalogen ether species showed similar associations with AD. Alkyl ether lipids are less susceptible to oxidation compared to their plasmenyl counterparts, suggesting associations with $\mathrm{AD}$ more likely reflect changes to the biosynthetic pathway. In support of this, Grimm et al. ${ }^{29}$ reported dysregulation of the peroxisomal enzymes relating to plasmalogen synthesis in $\mathrm{AD}$. Plasmalogens have been reported to be involved in several physiological functions including maintenance of lipid raft domain ${ }^{30}$ which are important for secretase function, responsible for $A \beta$ production ${ }^{31}$, cholesterol efflux ${ }^{32}$ and cellular survival ${ }^{33}$. Importantly, peripheral intravenous administration of plasmalogens has been shown to inhibit $A \beta$ accumulation in a study involving neuroinflammation $^{34}$. Impairment to the synthetic pathway of ether lipids may have deleterious downstream effects, and, in fact, these species have been proposed as potential therapeutic compounds ${ }^{35}$.

We have observed diverse associations of specific $n$-acylated ceramides with AD: positive associations were observed with species containing 18:0, 20:0 and 24:1 fatty acids, whereas negative or neutral associations were observed for species containing 22:0, 24:0 and 26:0 fatty acids, irrespective of the sphingoid base. In contrast, significant associations of sphingolipids with incident $\mathrm{AD}$ were only observed for species containing nervonic acid (24:1). This effect was much stronger in the AIBL study, while ADNI exhibited similar trends but were not significant after FDR correction. Despite this, the meta-analysis of sphingolipid associations resulted in much more power than utilising the AIBL study alone (Supplementary Table 3). 


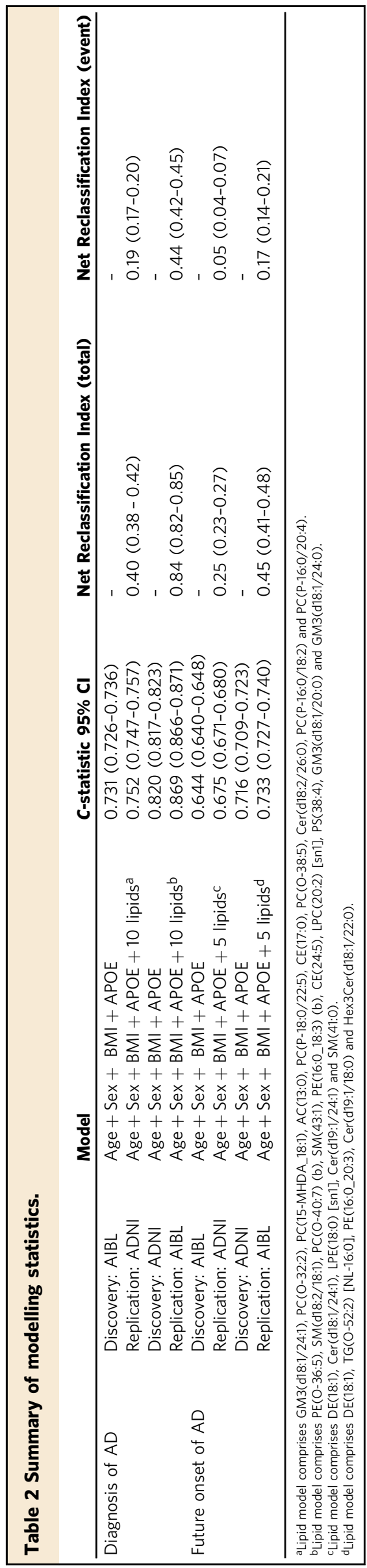

Synthesis of 24:1 is likely through elongation of 18:1 which itself is synthesised through unsaturation via stearoyl-CoA desaturase 1 (SCD-1). Interestingly, increased SCD-1 activity has previously been associated with $\mathrm{AD}^{36}$, and this may be contributing to the increased abundance of these monounsaturated lipid species. We observed similar positive associations with 18:1 relative to 18:0 in many of our measured lipid species, particularly species with a single fatty acid (e.g. LPC and CE species, Supplementary Table 3). Interestingly SCD-1 is associated with insulin resistance and adiposity, and mouse experiments have shown that disrupting SCD-1 function can potentially reduce body adiposity and improve insulin sensitivity ${ }^{37}$. This is relevant as obesity, insulin resistance and diabetes type II are all likely linked to increased risk of $\mathrm{AD}^{38}$

Specific ceramide synthases are responsible for the $n$-acylation of ceramide species, in particular, ceramide synthase 2 (20:0 to $26: 0)$ and $3(22: 0 \text { to } 26: 0)^{39}$. Decreases in the activity of ceramide synthase 2 (CerS2) have been observed in $\mathrm{AD}$, early in pathogenesis $^{40}$. Thus, the negative association of $\operatorname{Cer}(22: 0), \operatorname{Cer}(24: 0)$ and Cer(26:0) may be driven by decreases of CerS2, while the positive association of Cer(24:1) driven by SCD-1 cancels out the negative association observed with CerS2, which would, on its own, be expected to result in decreased levels of this species.

Gangliosides are a group of sphingolipids with oligosaccharide groups linked to the sphingoid base. $\mathrm{GM}_{3}$ gangliosides, the most abundant circulating ganglioside class, is positively associated with $\mathrm{AD}$. Gangliosides have been reported to accelerate $\mathrm{A} \beta$ aggregation, leading to deposition in the brain ${ }^{41}$. While the mechanism leading to increased circulating gangliosides is currently not known, gangliosides are not commonly measured, and thus far no other reports have described an association between circulating $\mathrm{GM}_{3}$ gangliosides and $\mathrm{AD}$.

The demographic and study design differences between the AIBL and ADNI cohorts presented a unique opportunity to validate some of the lipid markers in this study. Despite the geological differences with the two studies, the cross-sectional associations identify fairly similar associations and patterns within the lipidome and disease outcome. However, one challenge was the study design differences, where the distribution of control, MCI and AD cases were quite different (approximately 7:1:2.5 for AIBL and 1:2:1 for ADNI) which resulted in some differences of important variables in the multivariate modelling, thus requiring retraining of the coefficients in the replication set despite normalisation. Nonetheless, we were able to identify several lipids that appear to be key in identifying individuals who have, or are at, risk of developing AD. These lipids highlight unique features that provide information on top of easily obtained anthropometric (age, sex, BMI) and biochemical features ( $A P O E \& 4$ alleles). While we were able to test and validate these models across the two cohorts, ultimately a population study will be required to fully assess model performance.

While our lipidomic methodology offers a broad coverage of the lipidome, in the absence of stable isotope standards for each of the 569 lipid species, lipidomics will not provide exact quantification. We include a single internal standard per lipid class to provide quantitative data, while acknowledging that differential response factors will lead to slight offsets of the calculated concentration relative to their true concentration. However, this does not impact the association analyses performed in this study nor the multivariate modelling to predict prevalent and incident $\mathrm{AD}$.

Cross-sectional studies have some inherent limitations. We observed strong associations between 218 plasma lipid species and $\mathrm{AD}$. However, dysregulation of the lipidome can arise from sources other than the disease pathology (reverse causation), for example, dietary and activity changes arising as $\mathrm{AD}$ progresses. 
To address this, we were also able to perform association studies with incident $\mathrm{AD}$ where we identified similar, albeit weaker, associations with the majority of the same lipid species.

The AIBL and ADNI cohorts represent two powerful studies to characterise $\mathrm{AD}$ and the combination provides the opportunity to validate findings across studies. However, these cohorts were selected to be high risk for $\mathrm{AD}$ and so traditional risk factors, including age and APOE genotype, are stronger predictors than in an unselected population. As a consequence, these cohorts likely underestimate the performance of risk models for incident $\mathrm{AD}$ and further studies on balanced population cohorts will be required.

To conclude, we have performed one of the most comprehensive lipidomic analysis of $\mathrm{AD}$ to date by utilising two large, independent clinical studies in $\mathrm{AD}$. We have provided a holistic picture of lipid dysregulation associated with both prevalent and incident AD. Our plasma lipid dataset expands the scientific literature by providing greater resolution and allowing fine-granular analysis of the lipidome. Here we have highlighted specific changes to the ether lipid pathway, where plasmalogens are not the only drivers of ether lipid associations. Class-wide and species-specific changes highlight the necessity of a broad and detailed assay to capture these minute differences in the lipidome. We have demonstrated the potential of plasma lipids as the markers to improve assessment of prevalent and incident $\mathrm{AD}$, highlighting the importance of these small molecules in both disease prognostics and understanding the metabolic changes occurring with the disease.

\section{Methods}

Participants. The AIBL study recruited 1112 individuals over the age of 60 years into a longitudinal study ${ }^{42}$. At baseline, this comprised 768 cognitively normal, 133 with mild cognitive impairment and 211 with AD. Time points for blood/data collection were every 18 months from baseline. Detailed description of the participants was adapted from Ellis et al. ${ }^{42}$. We analysed all available fasted plasma samples (4106) from baseline up to the fifth time point. After filtering for missing values and problematic samples, the breakdown of sample numbers used in statistical analysis is presented in Table 1. In total, there were 1073, 963, 731, 702 and 564 samples at baseline, and follow-up times 1-4, respectively. While there were decreasing number of samples at successive time points, this was not completely driven by attrition, as some were missing owing to low sample material or were unobtainable at the start of the lipidomics study.

The ADNI1 study started in 2004 and recruited about 800 individuals at baseline. The initial goal was to recruit 200 participants with mild AD and 200 controls as well as 400 participants with MCI. Study data analysed here were obtained from the ADNI database, which is freely available online (http://adni.loni. usc.edu/). We utilised serum samples from the ADNI1 baseline cohort and followup data from the 6-, 12-, 18-, 24- and 36-month recalls for incident $\mathrm{AD}$ analysis.

Classification of disease state. Classification of MCI and AD in the AIBL cohort has been described extensively in previous publications ${ }^{42}$. Clinical criteria used to determine disease status included Mini Mental State Examination score of less than 28, failure on the Logical Memory test (in accordance with the ADNI criteria), other evidence of possible significant cognitive difficulty on neuropsychological testing, a Clinical Dementia Rating score of 0.5 or greater, a medical history suggestive of the presence of illnesses likely to impair cognitive function, an informant or personal history suggestive of impaired cognitive function, or who were consuming medications or other substances that could affect cognition ${ }^{42}$. A more indepth description of the ADNI cohort diagnostic criteria is reported elsewhere ${ }^{43}$; briefly, AD dementia diagnosis was established using NINDS-ADRDA criteria for probable AD. Classification as MCI followed the Petersen et al. ${ }^{44}$ criteria described previously.

Lipid extraction and liquid chromatography mass spectrometry. Lipids were extracted from $10 \mu \mathrm{l}$ plasma (AIBL) or serum (ADNI), with the addition of an internal standard mix (Supplementary Table 2), using the single-phase butanol/ methanol extraction method ${ }^{45}$. In brief, $10 \mu \mathrm{l}$ of samples were mixed with $100 \mu \mathrm{l}$ of $1: 1$ butanol:methanol containing the internal standards ${ }^{22}$, the samples were vortexed, sonicated on a sonicator bath and centrifuged $(13,000 \times g, 10 \mathrm{~min})$. The supernatants were transferred into glass vials and stored at $-80^{\circ} \mathrm{C}$. On average, 486 samples were extracted per day. Prior to mass spectrometry analysis, samples were thawed for $1 \mathrm{~h}$ at room temperature, vortexed and sonicated on the sonicator bath for $15 \mathrm{~min}$ and left to sit at $25^{\circ} \mathrm{C}$ for $2 \mathrm{~h}$ prior to analysis.
Analysis of plasma extracts was performed on an Agilent 6490 QQQ mass spectrometer with an Agilent 1290 series HPLC system. Mass spectrometry settings and transitions for each lipid class are shown in Supplementary Table 2.

Mass spectrometer running conditions were gas temperature $150^{\circ} \mathrm{C}$, gas flow rate $17 \mathrm{l} / \mathrm{min}$, nebulizer gas pressure $20 \mathrm{psi}$, sheath gas temperature $200^{\circ} \mathrm{C}$, capillary voltage $3500 \mathrm{~V}$ and sheath gas flow $10 \mathrm{l} / \mathrm{min}$. Isolation widths for Q1 and Q3 were set to "unit" resolution $(0.7 \mathrm{amu})$. The Agilent 1290 HPLC conditions were as follows: The composition of running solvents A and B comprised 50:30:20 and 1:9:90 water, acetonitrile and isopropanol, respectively. A ZORBAX eclipse plus $\mathrm{C} 18(2.1 \times 100 \mathrm{~mm} 1.8 \mathrm{~mm}$, Agilent $)$ column was used. Solvents were run at a flow rate of $0.4 \mathrm{ml} / \mathrm{min}$ with the column compartment temperature set to $60^{\circ} \mathrm{C}$. The solvent gradient was as follows: starting at $10 \% \mathrm{~B}$ and increasing to $45 \% \mathrm{~B}$ over $2.7 \mathrm{~min}$ (while diverting to waste for the first minute), then to $53 \% \mathrm{~B}$ over $0.1 \mathrm{~min}$, to $65 \%$ B over $6.2 \mathrm{~min}$, to $89 \%$ B over $0.1 \mathrm{~min}$, to $92 \%$ B over $1.9 \mathrm{~min}$ and finally to $100 \%$ over $0.1 \mathrm{~min}$. The solvent was then held at $100 \%$ B for $0.8 \mathrm{~min}$ and returned to $10 \% \mathrm{~B}$ over $0.1 \mathrm{~min}$ (a combined total of $12 \mathrm{~min}$ ). The column was reequilibrated in $10 \% \mathrm{~B}$ at an adjusted flow rate $(0.4 \mathrm{ml} / \mathrm{min}$ for $0.9 \mathrm{~min}$, then $0.6 \mathrm{ml} / \mathrm{min}$ for $1 \mathrm{~min}$, then back to $0.4 \mathrm{ml} / \mathrm{min}$ for $0.9 \mathrm{~min}$ ) prior to the next injection.

Additional experiments using pooled samples were utilised under varying conditions to get acyl composition data. To obtain further structural detail of isomeric and isobaric lipid species under the presented chromatographic conditions, we performed additional fragmentation experiments using pooled samples $^{22}$. The additional experiments to characterise the lipid species include acid hydrolysis, which selectively depletes plasmalogens leaving non-plasmalogen ether species intact, fragmentation in the presence of lithium ions in positive ionisation mode and fragmentation in negative ionisation mode, both of which yield characteristic product ions for structural elucidation under our reported analytical conditions ${ }^{22}$

Data integration, batch alignment and statistical analysis. Peak area of the lipid species was related to the internal standards to generate concentration data. To remove technical batch variation, the lipid data in each analytical batch (approximately 486 samples a batch) were aligned by using the median value of each lipid of the plasma quality control samples. As most lipid species were positively skewed, final lipid concentrations were $\log _{10}$ transformed prior to statistical analysis. Lipid class totals were generated by summing the individual species within each class.

All statistical analyses were performed in the R statistical platform (version 3.4.1+). For logistic and Cox regression, lipid data were scaled by the standard deviation and mean centred within each cohort. When examining associations with prevalent disease, we excluded MCI individuals from the analysis in both studies to provide a clean distinction between disease and non-disease. Furthermore, to maximise the number of $\mathrm{AD}$ samples in the prevalent disease analysis, we utilised the last acquired sample for each participants and have added covariates corresponding to each time point to adjust for possible confounding effects.

For examining incident disease analysis, we utilised the baseline samples only. This analysis included healthy and MCI individuals. For both analyses, the metaanalysis was performed using the "meta" package in $\mathrm{R}$ and was performed using a fixed effects model.

Correlation analysis was performed using Spearman correlation, with hierarchical clustering of co-linear lipids (complete distance method) followed by tree cutting using the Dynamic cut R package (hybrid method), resulting in 150 and 148 clusters for the AIBL and ADNI lipid data, respectively.

Common covariates utilised in statistical analysis include age, sex, BMI, HDL cholesterol, total cholesterol, clinical TGs, statin use and omega-3 supplementation FDR correction was performed using the method of Benjamini and Hochberg 46 using the nominal $p$ values from each univariate analysis (569 comparisons for individual lipid species and 32 comparisons for lipid classes). Meta-analysis to combine the results between the two cohorts was conducted using an inversevariance weighted averaged fixed effect meta-analysis, with similar FDR corrections applied to the nominal $p$ values. For modelling, each cohort was $Z$-scored independently.

Multivariate modelling of AD. We leveraged the availability of two independent cohorts to train and test different lipid models to diagnose prevalent disease (logistic models) and identify individuals at risk of future AD onset (Cox models). We used one cohort to define a set of features (discovery) for a multivariate model (common clinical variables + multiple lipid species) and used the second cohort to test these features for their predictive performance (replication). We then repeated this process switching the cohorts around.

With the AIBL cohort, we used a 2:1 age-sex-matched baseline subset (Supplementary Table 1) due to the low incidence of conversion and the large age difference between converters and non-converters within the cohort. Similarly, we did not adjust for MCI in these models to better allow the predictive performance of the lipidomic markers to be assessed. Due to the different population distribution and characteristics of the two cohorts (AIBL and ADNI) we retrained the model (using the selected features) on the replication cohort. This was also done in a cross-validated framework to avoid overfitting. 
To define a feature list in the discovery cohort, we generated a series of models using forward stepwise regression, adding lipid species to the existing covariates (to a maximum of 5 lipids for the Cox model and 10 for the logistic model), while minimising the Akaike Information Criterion, within a 10 -fold cross validation framework.

We rationalised that a stepwise approach would help us to maximise model performance while incorporating a minimum number of lipid species into the model, producing a simpler model to minimise the potential of overfitting. We used the frequency of incorporation into the training models, after accounting for clustering of co-linear lipids, to define a set of features that were subsequently validated in the replication cohort.

The need to consider highly correlated lipids within clusters in our selection process was due to the tendency of these lipid species to be selected in different cross-validation folds, and thus reduce the ranking of these correlated lipid species despite being strong predictors of AD. By considering these correlated lipids within clusters, ranking the clusters and selecting only the top ranked lipid within each cluster, we optimised the ranking of the most powerful predictors while avoiding the selection of highly correlated lipid species.

For replication, the features selected from the discovery cohort were modelled in the replication cohort, also within a 10 -fold cross-validation framework. The $C$ statistic and NRI were calculated after 200 repeats from the replication results. This process was repeated such that AIBL and ADNI were utilised as both discovery and replication sets.

Naming convention of lipid species. The lipid naming convention used here follows the guidelines established by the Lipid Maps Consortium and the shorthand notation established by Liebisch et al. ${ }^{47-49}$. We identified several species of interest that are not structurally resolved. These species separated chromatographically but incompletely characterised were labelled with an (a) or (b) to differentiate them, for example PC(P-17:0/20:4) (a) and PC(P-17:0/20:4) (b) where (a) and (b) represent the elution order. Separated isoforms that contain a 16:0 methyl branched fatty acid are presented as MHDA (methylhexadecanoic acid).

Reporting summary. Further information on research design is available in the Nature Research Reporting Summary linked to this article.

\section{Data availability}

Data to support these findings are available online and upon request. The entire ADNI lipidomic and clinical characteristic data are available online (adni.loni.usc.edu, specifically, ADMC Lipidomics Meikle Lab Baseline Data Matrix [ADNI1]) and the remaining data (AIBL) used in this study are available from both the corresponding authors and online at https://aibl.csiro.au upon reasonable request. Source data are provided with this paper.

Received: 19 August 2019; Accepted: 15 October 2020; Published online: 10 November 2020

\section{References}

1. Ferri, C. P. et al. Global prevalence of dementia: a Delphi consensus study. Lancet 366, 2112-2117 (2005).

2. Burla, B. et al. MS-based lipidomics of human blood plasma: a communityinitiated position paper to develop accepted guidelines. J. Lipid Res. 59, 2001-2017 (2018).

3. Goodenowe, D. B. et al. Peripheral ethanolamine plasmalogen deficiency: a logical causative factor in Alzheimer's disease and dementia. J. Lipid Res. 48, 2485-2498 (2007).

4. Igarashi, M. et al. Disturbed choline plasmalogen and phospholipid fatty acid concentrations in Alzheimer's disease prefrontal cortex. J. Alzheimer's Dis. 24, 507-517 (2011).

5. Mapstone, M. et al. Plasma phospholipids identify antecedent memory impairment in older adults. Nat. Med. 20, 415-418 (2014).

6. Han, X. L., Holtzman, D. M. \& McKeel, D. W. Plasmalogen deficiency in early Alzheimer's disease subjects and in animal models: molecular characterization using electrospray ionization mass spectrometry. J. Neurochem. 77, 1168-1180 (2001).

7. Cheng, H., Zhou, Y., Holtzman, D. M. \& Han, X. Apolipoprotein E mediates sulfatide depletion in animal models of Alzheimer's disease. Neurobiol. Aging 31, 1188-1196 (2010).

8. Yamamoto, N. et al. Accelerated Abeta aggregation in the presence of GM1ganglioside-accumulated synaptosomes of aged apoE4-knock-in mouse brain. FEBS Lett. 569, 135-139 (2004).

9. Han, X., Holtzman, D. M., McKeel, D. W., Kelley, J. \& Morris, J. C. Substantial sulfatide deficiency and ceramide elevation in very early Alzheimer's disease: potential role in disease pathogenesis. J. Neurochem. 82, 809-818 (2002).
10. Chatterjee, P. et al. Plasma phospholipid and sphingolipid alterations in Presenilin1 mutation carriers: a pilot study. J. Alzheimers Dis. 50, 887-894 (2016).

11. Whiley, L. et al. Evidence of altered phosphatidylcholine metabolism in Alzheimer's disease. Neurobiol. Aging 35, 271-278 (2014).

12. Jansen, I. E. et al. Genome-wide meta-analysis identifies new loci and functional pathways influencing Alzheimer's disease risk. Nat. Genet. 51 404-413 (2019).

13. Quehenberger, O. et al. Lipidomics reveals a remarkable diversity of lipids in human plasma. J. Lipid Res. 51, 3299-3305 (2010).

14. Fiandaca, M. S. et al. Plasma 24-metabolite panel predicts preclinical transition to clinical stages of Alzheimer's disease. Front. Neurol. 6, 237 (2015).

15. Li, D. et al. Plasma phospholipids and prevalence of mild cognitive impairment and/or dementia in the ARIC Neurocognitive Study (ARIC-NCS) Alzheimers Dement. 3, 73-82 (2016).

16. Koal, T., Klavins, K., Seppi, D., Kemmler, G. \& Humpel, C. Sphingomyelin SM (d18:1/18:0) is significantly enhanced in cerebrospinal fluid samples dichotomized by pathological amyloid-beta42, tau, and phospho-tau-181 levels. J. Alzheimers Dis. 44, 1193-1201 (2015).

17. Casanova, R. et al. Blood metabolite markers of preclinical Alzheimer's disease in two longitudinally followed cohorts of older individuals. Alzheimer's Dement. 12, 815-822 (2016)

18. Proitsi, P. et al. Association of blood lipids with Alzheimer's disease: a comprehensive lipidomics analysis. Alzheimers Dement. 13, 140-151 (2017)

19. Proitsi, P. et al. Plasma lipidomics analysis finds long chain cholesteryl ester to be associated with Alzheimer's disease. Transl. Psychiatry 5, e494 (2015).

20. Barupal, D. K. et al. Sets of coregulated serum lipids are associated with Alzheimer's disease pathophysiology. Alzheimer's Dement. 11, 619-627 (2019).

21. Lim, W. L. F. et al. Relationships between plasma lipids species, gender, risk factors, and Alzheimer's disease. J. Alzheimers Dis. 76, 303-315 (2020).

22. Huynh, K. et al. High-throughput plasma lipidomics: detailed mapping of the associations with cardiometabolic risk factors. Cell Chem. Biol. 26, 71-84.e4 (2018).

23. Meikle, P. J. et al. Plasma lipid profiling shows similar associations with prediabetes and type 2 diabetes. PLoS ONE 8, e74341 (2013).

24. Raffaitin, C. et al. Metabolic syndrome and risk for incident Alzheimer's disease or vascular dementia: the Three-City Study. Diabetes Care 32, 169-174 (2009).

25. Gudala, K., Bansal, D., Schifano, F. \& Bhansali, A. Diabetes mellitus and risk of dementia: a meta-analysis of prospective observational studies. J. Diabetes Investig. 4, 640-650 (2013).

26. Dorninger, F. et al. Homeostasis of phospholipids-the level of phosphatidylethanolamine tightly adapts to changes in ethanolamine plasmalogens. Biochim. Biophys. Acta 1851, 117-128 (2015).

27. Nagan, N. \& Zoeller, R. A. Plasmalogens: biosynthesis and functions. Prog. Lipid Res. 40, 199-229 (2001).

28. Goodenowe, D. B. et al. Peripheral ethanolamine plasmalogen deficiency: logical causative factor in Alzheimer's disease and dementia. J. Lipid Res. 48, 2485-2498 (2007)

29. Grimm, M. O. W. et al. Plasmalogen synthesis is regulated via alkyldihydroxyacetonephosphate-synthase by amyloid precursor protein processing and is affected in Alzheimer's disease. J. Neurochem. 116, 916-925 (2011).

30. Pike, L. J., Han, X. L., Chung, K. N. \& Gross, R. W. Lipid rafts are enriched in plasmalogens and arachidonate-containing phospholipids and the expression of caveolin does not alter the lipid composition of these domains. FASEB J. 15, A20 (2001).

31. Vetrivel, K. S. \& Thinakaran, G. Membrane rafts in Alzheimer's disease betaamyloid production. Biochim Biophys. Acta 1801, 860-867 (2010).

32. Mandel, $\mathrm{H}$. et al. Plasmalogen phospholipids are involved in HDL-mediated cholesterol efflux: insights from investigations with plasmalogen-deficient cells. Biochem. Biophys. Res. Commun. 250, 369-373 (1998).

33. Zoeller, R. A. et al. Increasing plasmalogen levels protects human endothelial cells during hypoxia. Am. J. Physiol. Heart Circ. Physiol. 283, H671-H679 (2002).

34. Ifuku, M. et al. Anti-inflammatory/anti-amyloidogenic effects of plasmalogens in lipopolysaccharide-induced neuroinflammation in adult mice. $J$. Neuroinflammation 9, 197 (2012).

35. Fujino, T. et al. Efficacy and blood plasmalogen changes by oral administration of plasmalogen in patients with mild alzheimer's disease and mild cognitive impairment: a multicenter, randomized, double-blind, placebocontrolled trial. EBioMedicine 17, 199-205 (2017).

36. Astarita, G. et al. Elevated stearoyl-CoA desaturase in brains of patients with Alzheimer's disease. PLoS ONE 6, e24777 (2011).

37. Ntambi, J. M. et al. Loss of stearoyl-CoA desaturase-1 function protects mice against adiposity. Proc. Natl Acad. Sci. USA 99, 11482-11486 (2002). 
38. Luchsinger, J. A., Tang, M.-X., Stern, Y., Shea, S. \& Mayeux, R. Diabetes mellitus and risk of Alzheimer' disease and dementia with stroke in a multiethnic cohort. Am J. Epidemiol. 154, 635-641 (2001).

39. Levy, M. \& Futerman, A. H. Mammalian ceramide synthases. IUBMB Life 62, 347-356 (2010).

40. Couttas, T. A. et al. Loss of ceramide synthase 2 activity, necessary for myelin biosynthesis, precedes tau pathology in the cortical pathogenesis of Alzheimer's disease. Neurobiol. Aging 43, 89-100 (2016).

41. Hoshino, T., Mahmood, M. I., Mori, K. \& Matsuzaki, K. Binding and aggregation mechanism of amyloid beta-peptides onto the GM1 gangliosidecontaining lipid membrane. J. Phys. Chem. B 117, 8085-8094 (2013).

42. Ellis, K. A. et al. The Australian Imaging, Biomarkers and Lifestyle (AIBL) study of aging: methodology and baseline characteristics of 1112 individuals recruited for a longitudinal study of Alzheimer's disease. Int. Psychogeriatr. 21, 672-687 (2009).

43. Weiner, M. W. et al. Impact of the Alzheimer's Disease Neuroimaging Initiative, 2004 to 2014. Alzheimers Dement. 11, 865-884 (2015).

44. Petersen, R. C. et al. Alzheimer's Disease Neuroimaging Initiative (ADNI): clinical characterization. Neurology 74, 201-209 (2010).

45. Alshehry, Z. H. et al. An efficient single phase method for the extraction of plasma lipids. Metabolites 5, 389-403 (2015).

46. Benjamini, Y. \& Hochberg, Y. Controlling the false discovery rate: a practical and powerful approach to multiple testing. J. R. Stat. Soc. Ser. B (Methodol.) 57, 289-300 (1995).

47. Fahy, E. et al. A comprehensive classification system for lipids. J. Lipid Res. 46, 839-861 (2005).

48. Fahy, E. et al. Update of the LIPID MAPS comprehensive classification system for lipids. J. Lipid Res. 50, S9-S14 (2009).

49. Liebisch, G. et al. Shorthand notation for lipid structures derived from mass spectrometry. J. Lipid Res. 54, 1523-1530 (2013).

\section{Acknowledgements}

Funding for the AIBL study was provided in part by the study partners [Commonwealth Scientific Industrial and research Organization (CSIRO), Edith Cowan University (ECU), Mental Health Research institute (MHRI), National Ageing Research Institute (NARI), Austin Health, CogState Ltd]. The AIBL study has also received support from the National Health and Medical Research Council (NHMRC) and the Dementia Collaborative Research Centres program (DCRC2), as well as funding from the Science and Industry Endowment Fund (SIEF) and the Cooperative Research Centre (CRC) for Mental Health-funded through the CRC Program (Grant ID:20100104), an Australian Government Initiative. This work was also supported in part by the Victorian Government's Operational Infrastructure Support Program. K.H. was supported by a Dementia Australia Research Foundation Scholarship. P.J.M. is supported by a Senior Research Fellowship from the National Health and Medical Research Council of Australia. Support for the metabolomics sample processing, assays and analytics reported here was provided by grants from the National Institute on Aging (NIA); NIA supported the Alzheimer's Disease Metabolomics Consortium which is a part of NIA's national initiatives AMP-AD and $\mathrm{M}^{2} \mathrm{OVE}-\mathrm{AD}$ (R01 AG046171, RF1 AG051550, RF1 AG057452 and 3U01 AG024904-09S4). Additional NIH support from the NIA, NLM and NCI for analysis includes P30 AG10133, R01 AG19771, R01 LM012535, R03 AG054936, R01 AG061788, K01 AG049050 and R01 CA129769. M.A. is supported by National Institute on Aging grants RF1 AG057452, RF1 AG058942, RF1 AG059093 and U01 AG061359. M.A. is also supported by funding from Qatar National Research Fund NPRP8-061-3-011. K.N. is supported by NLM R01 LM012535 and NIA R03AG054936. Data collection and sharing for the ADNI was supported by National Institutes of Health Grant U01 AG024904. ADNI is funded by the National Institute on Aging, the National Institute of Biomedical Imaging and Bioengineering, and through generous contributions from the following: AbbVie, Alzheimer's Association; Alzheimer's Drug Discovery Foundation; Araclon Biotech; BioClinica, Inc.; Biogen; Bristol-Myers Squibb Company; CereSpir, Inc.; Cogstate; Eisai Inc.; Elan Pharmaceuticals, Inc.; Eli Lilly and Company; EuroImmun; F. Hoffmann-La Roche Ltd and its affiliated company Genentech, Inc.; Fujirebio; GE Healthcare; IXICO Ltd; Janssen Alzheimer Immunotherapy Research \& Development, LLC; Johnson \& Johnson Pharmaceutical Research \& Development LLC; Lumosity; Lundbeck; Merck \& Co., Inc.; Meso Scale Diagnostics, LLC; NeuroRx Research; Neurotrack Technologies; Novartis Pharmaceuticals Corporation; Pfizer Inc.; Piramal Imaging; Servier; Takeda Pharmaceutical Company; and Transition Therapeutics. The Canadian Institutes of Health Research is providing funds to support ADNI clinical sites in Canada. Private sector contributions are facilitated by the Foundation for the National Institutes of Health (www.fnih.org). The grantee organization is the Northern California
Institute for Research and Education, and the study is coordinated by the Alzheimer's Therapeutic Research Institute at the University of Southern California. ADNI data are disseminated by the Laboratory for Neuro Imaging at the University of Southern California. This study was only possible with the help of the AIBL research group. The authors who made direct contribution to this study have been listed as authors in this article. Members of the AIBL group who did not participate in the analysis or writing of this report are listed here: https://aibl.csiro.au/about/aibl-research-team/. Part of the data used in preparation of this article were obtained from the Alzheimer's Disease Neuroimaging Initiative (ADNI) database (adni.loni.usc.edu). The authors who made direct contribution to this study have been listed as authors in this article. As such, the investigators within the ADNI contributed to the design and implementation of ADNI and/or provided data but did not participate in analysis or writing of this report. A complete listing of ADNI investigators can be found at: http://adni.loni.usc.edu/wpcontent/uploads/how_to_apply/ADNI_Acknowledgement_List.pdf. Part of the data used in preparation of this article were generated by the Alzheimer's Disease Metabolomics Consortium (ADMC). The authors who made direct contribution to this study have been listed as authors in this article. Investigators within the ADMC provided data but did not participate in analysis or writing of this report can be found at https://sites.duke.edu/ adnimetab/team/.

\section{Author contributions}

P.J.M., R.N.M. and R.K.-D. conceived and designed this study. K.H., C.G., W.L.F.L. and N.A.M. handled the samples and did the mass spectrometry analysis. K.H. and W.L.F.L. analysed the mass spectrometry results. K.H., C.G., K.S.J., A.S., A.A.T. and G.O. performed the statistical analysis and provided interpretation of results. P.C., I.M., S.M.L., A.I.B., C.C.R., V.L.V., D.A. and C.L.M. provided clinical data and support for the AIBL study. M.A., K.N., A.J.S., R.B., X.H. and R.K.-D. provided clinical data and support for the ADNI study. P.J.M. and B.G.D. supervised K.H. in developing the lipidomics platform used in this study. P.J.M. and R.N.M. provided funding to perform initial analysis of the AIBL study, P.J.M. and R.K.-D. provided funding to perform initial analysis of the ADNI study. All authors have reviewed, edited and provided feedback on the manuscript.

\section{Competing interests}

P.J.M., R.N.M., K.H. and W.L.F.L filed a provisional patent using the AIBL data Application number: App Number 2018901220; DEMENTIA RISK ANALYSIS; Baker Heart and Diabetes Institute, Edith Cowan University. All other authors declare no competing interests.

\section{Additional information}

Supplementary information is available for this paper at https://doi.org/10.1038/s41467 020-19473-7.

Correspondence and requests for materials should be addressed to R.K.-D., R.N.M. or P.J.M.

Peer review information Nature Communications thanks Petroula Proitsi and other, anonymous, reviewers for their contributions to the peer review of this work.

Reprints and permission information is available at http://www.nature.com/reprints

Publisher's note Springer Nature remains neutral with regard to jurisdictional claims in published maps and institutional affiliations.

Open Access This article is licensed under a Creative Commons Attribution 4.0 International License, which permits use, sharing, adaptation, distribution and reproduction in any medium or format, as long as you give appropriate credit to the original author(s) and the source, provide a link to the Creative Commons license, and indicate if changes were made. The images or other third party material in this article are included in the article's Creative Commons license, unless indicated otherwise in a credit line to the material. If material is not included in the article's Creative Commons license and your intended use is not permitted by statutory regulation or exceeds the permitted use, you will need to obtain permission directly from the copyright holder. To view a copy of this license, visit http://creativecommons.org/ licenses/by/4.0\%

(C) The Author(s) 2020 